\title{
Potential application of GSTT1-null genotype in predicting toxicity associated to 5-fluouracil irinotecan and leucovorin regimen in advanced stage colorectal cancer patients
}

\author{
R. ZÁRATE ROMERO ${ }^{1}$, R. MORALES ${ }^{2}$, F. GARCIA ${ }^{1}$, M. HUARRIZ ${ }^{1}$, E. BANDRES ${ }^{1}$, \\ J. DE LA HABA ${ }^{2}$, A. GÓMEZ ${ }^{2}$, E. ARANDA ${ }^{2}$ and J. GARCÍA-FONCILLAS ${ }^{1}$ \\ ${ }^{1}$ Laboratory of Biotechnology and Pharmacogenomics, Center for Applied Medical Research, \\ University Clinic of Navarra, 36 Pio XII Ave., Navarra 31008; ${ }^{2}$ Oncologic Medical Service, \\ University Hospital Reina Sofía of Córdoba, Menéndez Pidal Ave., Córdoba 14004, Spain
}

Received March 10, 2006; Accepted May 18, 2006

\begin{abstract}
Our aim was to evaluate the role of C-69T in GSTA1, Ile105Val in GSTP1, null allele in GSTT1 and GSTM1 in the prediction of toxicity in patients treated with 5-Fu/CPT-11/Lv regimens in metastatic CRC patients. Fiftyone patients with CRC metastatic disease were analysed. All patients had bidimensionally measurable disease according to WHO criteria. The gender distribution was 37 (74\%) males and $13(26 \%)$ females; age ranged from 41 to 71 years; performance status was in all patients $\geq 80$ (Karnofsky index). The analysis of gene polymorphism was performed in lymphocytes by using PCR-RFLP (GSTA1, GSTP1), PCR (GSTT1, GSTM1) and sequencing analysis (UGT1A1*28). An appreciable significant association was observed between the GSTT1-null and toxicity: $57 \%$ developed gastrointestinal toxicity grade III versus $23 \%$ of patients with GSTT1-present genotype $(\mathrm{p}=0.053)$. The other polymorphisms analysed did not show any significant relation with toxicity. Our data suggest that GSTT1-null is associated with a greater probability of developing toxicity to 5 -Fu/CPT-11/Lv treatments, indicating a potential application of this genetic analysis in predicting adverse effects of this regimen.
\end{abstract}

\section{Introduction}

5-Fluouracil (5-FU) has been the mainstay of treatment for patients with advanced colorectal cancer (CRC) for more than five decades. Irinotecan (CPT-11) has been found to

Correspondence to: Dr R.N. Zárate Romero or Dr J. GarcíaFoncillas, Laboratory of Biotechnology and Pharmacogenomics, Center for Applied Medical Research, University Clinic of Navarra, 36 Pio XII Ave., Navarra 31008, Spain

E-mail: rzarate@unav.es; jgfoncillas@unav.es

Key words: colorectal cancer, glutathione S-transferase, polymorphism, toxicity demonstrate at least equivalent efficacy to 5-FU in first-line therapy, favorable quality-of-life assessments and prolonged median survival $(1,2)$. In Europe as well as in the USA, the combination of 5-FU plus CPT-11 is currently recommended as first-line therapy for metastatic CRC treatment $(3,4)$. Risk factors with predictive value for toxicity have been identified in several studies. In this sense, age, performance status, bilirubinaemia, the genetic polymorphism of UDP-glucuronyltransferase-1A1 (UGT1A1) and the drug administration schedule have been show to be related with CPT-11 toxicity (5). Inter-individual differences in the pharmacokinetics of its active metabolite, $\mathrm{SN}-38$, cause the variations in the effect of the drug (6). Several studies in relation with different doses and schedules of CPT-11 alone or in combination with other agents are ongoing to investigate its use as first or secondline therapy $(7,8)$, with a view to optimising the therapeutic outcome for these patients.

On the other hand, Glutathione S-transferases (GSTs) are considered an important family of detoxifying enzymes for mutagens. They protect cellular macromolecules from damage by catalysing the conjugation of toxic and carcinogenic electrophilic molecules with glutathione. The resulting complex is less toxic and more readily excreted.

The implication of GSTs in the detoxification of heterocyclic amines (HCAs) and polycyclic aromatic hydrocarbons (PAHs) and other carcinogens has been related to GST gene alterations with colorectal cancer risk $(9,10)$. Diverse common single nucleotide polymorphisms (SNPs) have been reported for GSTT1, GSTM1, GSTA1 and GSTP1 genes that either abolish, increase or decrease these enzyme activities (11). Homozygous inherited deletions in the GSTM1 and GSTT1 gene have been related with the reduced detoxification capacity and increased genotoxic susceptibility (12). Differential GSTT1 and GSTM1 expression have been determined in erythroid and lymphoid cells, respectively in cancer study (13). Moreover, these proteins have been localized also in the liver, the major site of drug metabolism, and colon tissue, although the expression of GSTM1 in the colon is lower compared to the liver. GSTP1 has a high expression level in the colon but is a minor component in the liver (14). The changed polymorphism Ile105Val, modifies 
the enzyme affinity and activity for electrophilic substances. GSTA1 is expressed at higher levels in the liver and one single nucleotide change (C-69T) in the proximal promoter reduced expression and activity of this gene (10). GSTP1 is linked to oxidative damage to nucleic acid; whereas, GSTA1 and GSTM1 are associated to lipid peroxidation (15).

Inter-individual differences in cancer susceptibility may be mediated in part through polymorphic variability in the bio-activation and detoxification of carcinogens. In this context, GSTs genotype may influence individual states through its implications in detoxification of treatment agents, GSTmediated protection against oxidative damage during treatment and the differences in carcinogen damage to DNA. Several reports have related GSTs consistently as cancer susceptibility genes $(9,16,17)$ and few studies report the toxicity relation of GSTs with chemotherapeutic agents in CRC.

The possibility of individualizing cancer treatment is gaining wide acceptance. In the present study we evaluated influence and possible relation between common null-alleles in both GSTT1 and GSTM1 genes and SNPs in GSTP1 (I105V), GSTA1 (C-69T) and the UGT1A1*28 variant respect to toxicity in patients with metastatic CRC treated with a $5-\mathrm{Fu} / \mathrm{CPT}-11$ schedule.

\section{Materials and methods}

Patients. Fifty-one patients treated at the Universitary Hospital Reina Sofía, Córdoba, Spain, were included in this study. All patients had been diagnosed stage IV colorectal cancer and received 5-Fu/CPT-11/Lv regimens. All patients had bidimensionally measurable disease (according to WHO criteria) at the time of starting the treatment. Ascites and pleural effusions were not considered measurable. The gender distribution was, $38(75 \%)$ males and $13(25 \%)$ females with a median age of 63 (range 41-71 years). Ten patients (19\%) developed a tumour in the right colon, $16(31 \%)$ in the transverse and left colon and $25(49 \%)$ in the rectum. Of the patients $83 \%$ presented multiple metastases, of which $57 \%$ in the liver, and $47 \%$ had received previous adjuvant chemotherapy. The performance status (PS) was classified according to Karnofsky Index (KI). The most frequent PS in patients enrolled in this study was $100(73 \%)$, range 80-100 (Table I). All patients provided informed written consent before participating in our study.

Treatment schedule. Treatment was administered in the following schedule: $5-\mathrm{Fu} 2.250 \mathrm{mg} / \mathrm{m}^{2} 48 \mathrm{~h}$ in continuous infusion (CI) and $180 \mathrm{mg} / \mathrm{m}^{2}$ in $30 \mathrm{~min}$ of CPT-11, both every 14 days (Digestive Tumour Treatment regimen). The median number of cycles was 5 .

Evaluation criteria. Physical examinations and blood counts were performed after each chemotherapy cycle. Hepatic and renal function tests and computed-tomography (CT) scans of measurable lesions were assessed at baseline and repeated every 3 months.

Toxicity. Treatment toxicity was assessed before each cycle using the National Cancer Institute Common Toxicity Criteria (NCI-CTC). We defined 'severe toxicity' as haematological or gastrointestinal toxicity of grade III-IV. If neutrophils
Table I. Patient characteristics.

\begin{tabular}{|c|c|}
\hline Patients & Total \\
\hline \multicolumn{2}{|l|}{ Sex } \\
\hline Male & 38 \\
\hline Female & 13 \\
\hline \multicolumn{2}{|l|}{ Age (years) } \\
\hline Median & 63 \\
\hline Range & $41-77$ \\
\hline \multicolumn{2}{|l|}{$\begin{array}{l}\text { Karnofsky performance } \\
\text { status }(\%)\end{array}$} \\
\hline 100 & 37 \\
\hline 90 & 12 \\
\hline 80 & 2 \\
\hline \multicolumn{2}{|l|}{ Primary tumour location } \\
\hline Right colon & 10 \\
\hline Transverse and left colon & 16 \\
\hline Rectum & 25 \\
\hline \multicolumn{2}{|c|}{ Previous adjuvant chemotherapy } \\
\hline Yes & 24 \\
\hline No & 27 \\
\hline \multicolumn{2}{|l|}{ Metastasis } \\
\hline Single & 8 \\
\hline Multiple & 43 \\
\hline \multicolumn{2}{|l|}{ Liver metastasis } \\
\hline Yes & 29 \\
\hline No & 21 \\
\hline
\end{tabular}

$<1.5 \times 10^{9} / 1$, platelets $<100 \times 10^{9} / 1$ were observed or if there was significant persisting non-haematological toxicity, chemotherapy was delayed until all manifestations of toxicity had disappeared. We reduced doses for chemotherapy by $20 \%$ if severe toxic effects appeared.

Supportive care included intensive treatment with loperamide for late diarrhea. Atropine was given as needed for CPT-11-related cholinergic symptoms. An antiemetic agent was provided at the discretion of the treating physician.

Treatment discontinuation. Treatment was given until disease progression, the appearance of unacceptable toxicity or patient refusal.

Genotyping. Genomic DNA was extracted from $200 \mu 1$ of whole blood using the DNA Isolation Kit I from MagNa Pure LC (Roche, Barcelona, Spain) according to the protocols and software provided by the company.

GSTA1 and GSTP1 polymorphisms: Briefly, $10 \mu 1$ of the genomic DNA (300 ng) was used as a template with $200 \mathrm{ng}$ of primers, forward (5'-AGAATCCAGTAGGTGGCCCC-3') and reverse (3'-TGTTAAACGCTGTCACCGTCC-5') by 
A.

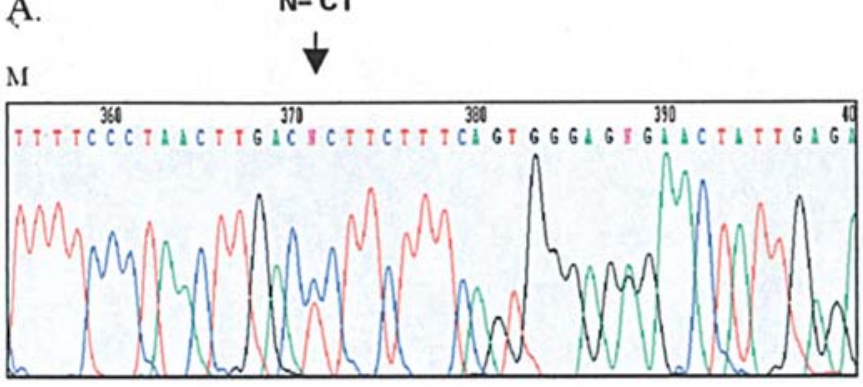

B.

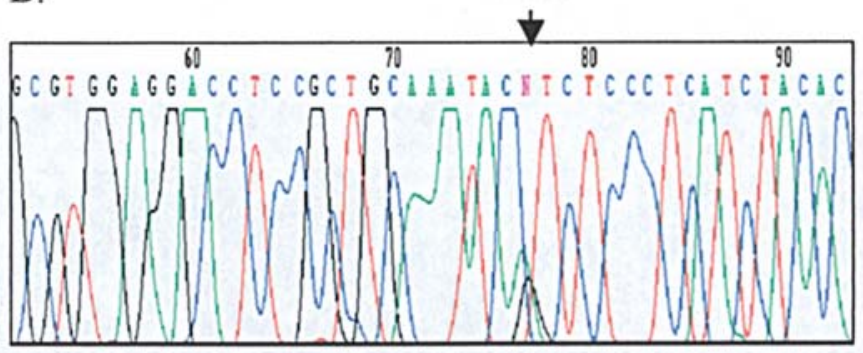

CT TT $\quad \mathrm{CC} \quad \mathrm{CC}$
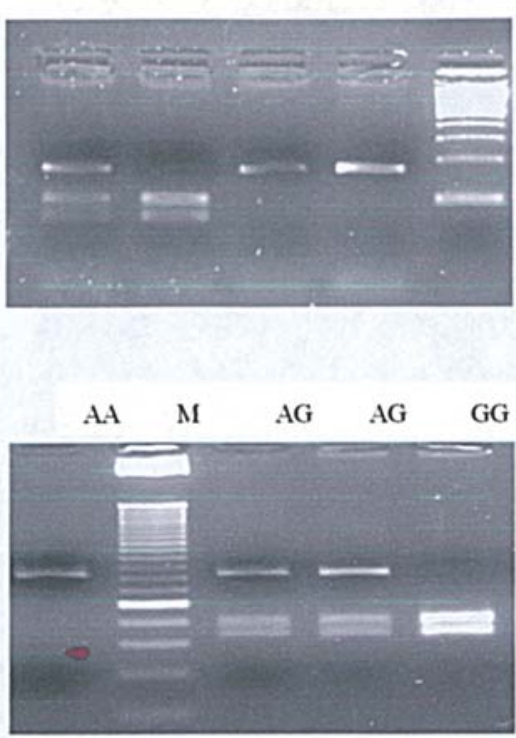

C.

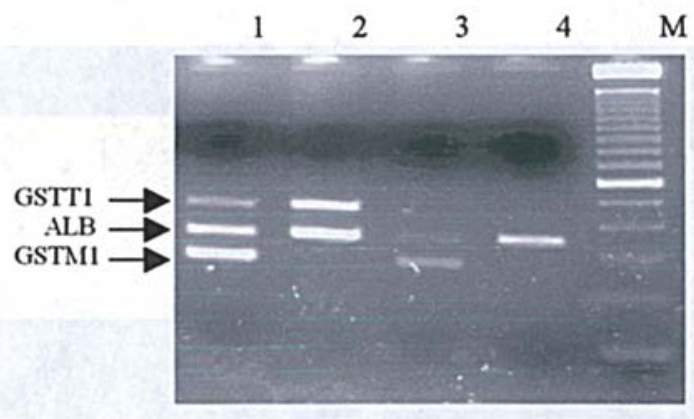

D.

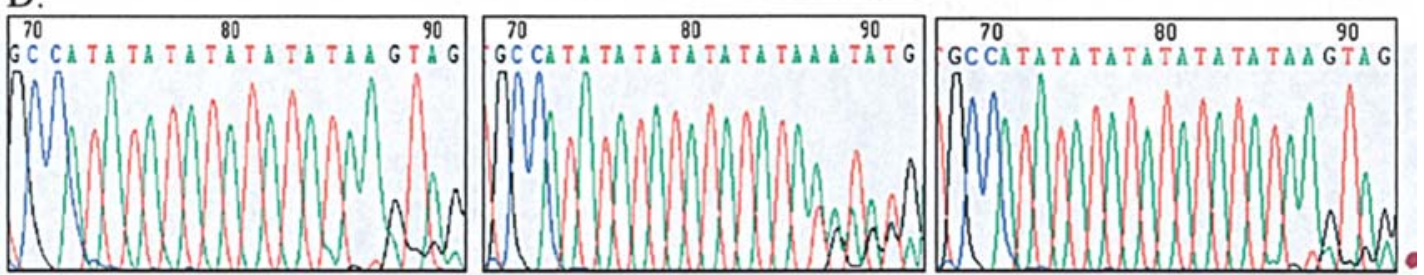

Figure 1. Digestion product. (A), GSTA1 genotype profile: heterozygote (CT), variant (TT) and wild-type (CC), and (B) GSTP1 wild-type (AA), heterozygote allele (AG) and variant (GG). (C), GSTT1 and GSTM1 allele analyses. Line 1, GSTT1 and GSTM1 present alleles. Line 2 shows the GSTT1 allele and homozygous deletion of GSTM1 and vice versa in line 3. Last in line 4 both GSTT1 and GSTM1 alleles are null. The molecular weight marker (M) used in (A) and (C) is of $100 \mathrm{bp}$; and $25 \mathrm{bp}$ in (B). (D), Automated sequencing of the A(TA)nTAA motif, in the promoter region of the UGT1A1 gene with homozygous 6/6 (left) heterozygous 6/7 (center) and homozygous 7/7 TA repeats (right).

GSTA1 amplicon (164 bp), designed with primer express 2.0 software (Applied Biosystems, Madrid, Spain). The GSTP1 fragment polymorphism (177 bp) was analysed using primers described by Harries et al (18). The PCR mix containing 2.5 U AmpliTaq Gold ${ }^{\mathrm{TM}}$ (Applied Biosystems) and the rest of the PCR components in a total volume of $50 \mu 1$.

The polymorphism regions were analysed by restriction fragment length polymorphism (RFLP) technique. Digestions were carried out in a final volume of $50 \mu 1$, containing $20 \mu 1$ of PCR product. Appropriate units of EarI and BsmAI enzymes were added to the other components provided by New England Biolabs (NEB, Beverly, MA, USA) for GSTA1 and GSTP1 product amplified, respectively. The amplicon digestion products were analysed in an LM-Sieve $3 \%$ agarose gel (Pronadisa, Madrid, Spain) stained with ethidium bromide and visualized by UV-induced fluorescence (Fig. 1A and $\mathrm{B}$ ).

GSTT1 and GSTM1 polymorphisms. The presence or absence (null genotype) of the GSTT1 (459 bp) or GSTM1 (273 bp) 
genes was determined simultaneously using allele-specific sequence primers through a multiplex PCR protocol, according to the method of Kim et al (19). The absence of amplification products was consistent with the null genotypes (homozygous deletion). Control primers that amplify albumin gene (350 bp) were also included in each reaction to confirm the presence of amplifiable DNA in the samples (Fig. 1C). The resulting amplicons were separated on a $2 \%$ agarose gel (Pronadisa).

Analysis of the A(TA)nTAA motif in the promoter region of the UGT1A1 gene was performed by PCR, according to Monaghan et al (20), followed by automated sequencing of the purified PCR product (Fig. 1D).

Quality control. Each PCR was realized with a negative control (without DNA) to test for possible contamination. Moreover, the GSTT1 and GSTM1 study used the albumin gene (ALB) as an internal positive control. When one of these controls failed the PCR was repeated.

The GSTA1 and GSTP1 amplicon were completely sequenced to confirm the genotype observed by RFLP technique. Direct sequencing of the purified PCR products was performed on an ABI PRISM ${ }^{\mathrm{TM}} 377$ DNA Sequencer by BigDye Terminator cycle sequencing reactions, ABI PRISM 377XL collection, DNA Sequencing Analysis 3.4.1, and Sequence Navigator software (Applied Biosystems).

Statistical analysis. Statistical analysis was performed with SPSS software 11.0 version for Windows (SPSS, Inc., Chicago, IL, USA). The $\chi^{2}$ tests for contingency tables were used for statistical analysis. The level of statistical significance was set-up at $\mathrm{p} \leq 0.05$.

The relationship between any of GSTs genotypes with toxicity and other clinical or pathological characteristics was assessed with the maximun likelihood $(L R)$ test, by stepwise method.

\section{Results}

Toxicity analysis related with GSTs, UGT1A1*28 polymorphisms. Individuals with GSTT1 were more frequent than GSTT1-null individuals (0.86 vs. 0.14 , respectively). In contrast, a similar frequency was observed in GSTM1 and null gene (0.41 and 0.59 , respectively). Four patients $(8 \%)$ showed deletion of both genes.

A significantly increased proportion of GSTT1-null genotype was detected in women $(\mathrm{p}=0.002)$ and GSTM1-null in men $(\mathrm{p}=0.01)$ (Table II). Moreover, significant association was observed with GSTT1-null genotype, 57\% (4/7) digestive toxicity grade III versus $23 \%(10 / 44)$ of patients with GSTT1-present genotype, $(\mathrm{p}=0.053)$, (Table III). However, after the $L R$ test, the toxicity was independent of the patient gender and the relationship with the GSTT1-null was maintained.

Respect to grade III/IV toxicities, the most frequent toxicity was diarrhoea $23.5 \%(12 / 51)$. Other toxicities are shown in Table IV. However, they were put together for the analysis of grade III/IV toxicities (Yes/No), joining the different haematological (anaemia, thrombocytopenia, neutropenia and febrile neutropenia) and gastrointestinal toxicities (diarrhea, mucositis and vomiting). We have to point out that $45 \%$
Table II. Genotypes and gender frequency.

\begin{tabular}{|c|c|c|c|}
\hline \multirow[t]{2}{*}{ Genotype } & \multicolumn{2}{|c|}{ Gender } & \multirow[t]{2}{*}{ P-value } \\
\hline & Male & Female & \\
\hline GSTT1 & \multicolumn{2}{|c|}{ (Genotype \%) } & 0.002 \\
\hline Present & $36(82)$ & $8(18)$ & \\
\hline Null & $2(29)$ & $5(71)$ & \\
\hline GSTM1 & & & 0.01 \\
\hline Present & $12(57)$ & $9(43)$ & \\
\hline Null & $26(87)$ & $4(13)$ & \\
\hline GSTA1 & & & 0.6 \\
\hline $\mathrm{CC}$ & $11(73)$ & $4(27)$ & \\
\hline $\mathrm{CT}$ & $18(82)$ & $4(18)$ & \\
\hline $\mathrm{TT}$ & $9(64)$ & $5(36)$ & \\
\hline GSTP1 & & & 0.2 \\
\hline AA & $15(65)$ & $8(35)$ & \\
\hline $\mathrm{AG}$ & $20(83)$ & $4(17)$ & \\
\hline GG & $3(75)$ & $1(25)$ & \\
\hline UGT1A1 & & & 0.5 \\
\hline $6 / 6$ & $15(71)$ & $6(29)$ & \\
\hline $6 / 7$ & $19(73)$ & 7 (27) & \\
\hline $7 / 7$ & $3(100)$ & 0 & \\
\hline
\end{tabular}

(23/51) of the patients developed grade III toxicity (Table IV). The overall distribution of gastro-intestinal and haematological toxicity was $78 \%(14 / 18)$ and $22 \%$ (4/18), respectively.

We have also considered other toxicities that are not related to the groups mentioned above (e.g. alopecia, nail and cardiac toxicity). Treatment with 5-FU was suspended in the case of the patient with cardiac toxicity and the symptomatology disappeared.

The UGT1A 1*28 heterozygous and homozygous conditions were most frequent in men, but without statistical significance. No relationship existed between the UGT1A1*28 condition and gastrointestinal or haematological toxicities (Table III).

Other clinical or pathological (special number of metastasis and tumour location) characteristics were not significantly associated with these polymorphic genes (data not shown).

\section{Discussion}

Two important points must be considered with respect to therapeutic outcome for patients with advanced colorectal cancer (CRC): control of the disease and quality of life where the secondary effects of the chosen treatment are under control. The step from monotherapy to polychemotherapy has had a positive effect, reflected both in the increase of the response rate and also in survival and time to progression (3). Nonetheless, the increase in toxicity has been a limiting factor, manageable only in certain treatment schedules (21). The key to this progress seems to lie in the introduction of 
Table III. Gastrointestinal $(n=14)$ and haematological $(n=4)$ grade III toxicity in GSTs and UGT1A1 analysed.

\begin{tabular}{|c|c|c|c|c|c|}
\hline & & AA & $\mathrm{AG}$ & GG & P-value \\
\hline \multirow[t]{3}{*}{ GSTP1 } & Gastro-intestinal & $7 / 23(30 \%)$ & $7 / 24(29 \%)$ & - & 0.4 \\
\hline & Haematological & $1 / 23(4.3 \%)$ & $3 / 24(13 \%)$ & - & 0.4 \\
\hline & & $\mathrm{CC}$ & CT & TT & \\
\hline \multirow[t]{3}{*}{ GSTA1 } & Gastro-intestinal & $4 / 15(27 \%)$ & $6 / 22(27 \%)$ & $4 / 14(29 \%)$ & 0.9 \\
\hline & Haematological & $1 / 15 \quad(7 \%)$ & $3 / 22(14 \%)$ & - & 0.3 \\
\hline & & \multicolumn{2}{|c|}{ PRESENT } & NULL & \\
\hline \multirow[t]{3}{*}{ GSTT1 } & Gastro-intestinal & \multicolumn{2}{|c|}{$10 / 44(23 \%)$} & $4 / 7 \quad(57 \%)$ & $0.053^{\mathrm{a}}$ \\
\hline & Haematological & \multicolumn{2}{|c|}{$4 / 44(9 \%)$} & - & 1 \\
\hline & & \multicolumn{2}{|c|}{ PRESENT } & NULL & \\
\hline \multirow[t]{3}{*}{ GSTM1 } & Gastro-intestinal & \multicolumn{2}{|c|}{$5 / 21(24 \%)$} & $9 / 30(30 \%)$ & 0.4 \\
\hline & Haematological & \multicolumn{2}{|c|}{$2 / 21(10 \%)$} & $2 / 30 \quad(7 \%)$ & 0.5 \\
\hline & & $6 / 6$ & $6 / 7$ & $7 / 7$ & \\
\hline \multirow[t]{2}{*}{ UGT1A1 ${ }^{*} 28$} & Gastro-intestinal & $6 / 21(29 \%)$ & $8 / 26(31 \%)$ & - & 0.4 \\
\hline & Haematological & $2 / 21(10 \%)$ & $2 / 26 \quad(8 \%)$ & - & 0.8 \\
\hline
\end{tabular}

${ }^{\text {aP-value }} \leq 0.05$.

Table IV. Overall treatment-related toxicity, graded according to the NCI-CTC $(n=51)$.

\begin{tabular}{|c|c|c|c|c|c|c|}
\hline \multirow[t]{2}{*}{ Toxicity } & \multicolumn{5}{|c|}{ Grade } & \multirow[t]{2}{*}{ Total grade III-IV (\%) } \\
\hline & 0 & I & II & III & IV & \\
\hline Neutropenia & 34 & 5 & 10 & 2 & 0 & $2 \quad(3.9)$ \\
\hline Vomiting & 23 & 13 & 13 & 2 & 0 & $2 \quad(3.9)$ \\
\hline Diarrheoa & 17 & 8 & 14 & 12 & 0 & $12(23.5)$ \\
\hline Mucositis & 35 & 8 & 6 & 2 & 0 & $2 \quad(3.9)$ \\
\hline Constipation & 39 & 11 & 1 & 0 & 0 & 0 \\
\hline Hepatic & 51 & 0 & 0 & 0 & 0 & 0 \\
\hline Cardiac & 49 & 0 & 1 & 1 & 0 & $1 \quad(1.9)$ \\
\hline Anemia & 37 & 13 & 0 & 1 & 0 & $1 \quad(1.9)$ \\
\hline Alopecia & 44 & 1 & 4 & 2 & 0 & $2 \quad(3.9)$ \\
\hline Febrile neutropenia & 50 & 0 & 0 & 1 & 0 & $1 \quad(1.9)$ \\
\hline Thrombocytopenia & 51 & 0 & 0 & 0 & 0 & 0 \\
\hline Anorexia & 46 & 4 & 1 & 0 & 0 & 0 \\
\hline Cutaneus & 48 & 2 & 1 & 0 & 0 & 0 \\
\hline Ocular & 49 & 2 & 0 & 0 & 0 & 0 \\
\hline Asthenia & 34 & 13 & 4 & 0 & 0 & 0 \\
\hline
\end{tabular}

new drugs in combination with 5-FU, such as CPT-11 or oxaliplatin.

However, it should be noted that heterogeneity in the efficacy and toxicity of chemotherapeutic agents has been observed across the human population. Administration of the same dose of an anticancer drug to a group of patients results in a range of toxicity, from the unaffected to lethal events.
Published studies report that the toxicities due to CPT-11 are primarily neutropenia and diarrhoea (22-24). In this sense, patients with the UGT1A1*28 allele (7/7 TA repeat or more) may development this toxicity easily after CPT-11 chemotherapy. In our treatment schedule, this relation was not observed. In contrast, there is a direct GSTT1 implication in toxicity development with an appreciable significant relation 
between the GSTT1-null gene and general and gastro-intestinal toxicity. Our results are consistent with some recent studies but in acute myeloid leukaemia (AML) suggesting that the lack of the GSTT1 gene implies greater toxicity in these patients (25-27). Similar observations have been made in lung cancer (28). The chemotherapy agent employed in these studies are different to ours, however, determining which drug's metabolism might be influenced by GSTT1 genotype is difficult as the substrate specificity remains unclear. Genotoxic effects of the agent diepoxybutane in GSTT1-null condition and the environmental carcinogen benzene have been reported in in vitro sensitivity assay (29-31).

The patients included in this study group were selected according to type, tumoural stage and treatment schedule and the frequency determined for the GSTT1 and GSTM1-null ( 0.14 and 0.59 , respectively) gene was similar to other studies realized in Caucasian CRC groups (32-34), as well as in other European countries (GSTT1-null 0.13 in Finnish and Swedish populations and 0.58 for GSTM1-null in Portuguese populations). Although in contrast with that indicated for Spanish populations, where the loss of both genes had slightly lower frequency (35).

In our patients, the proportion of GSTT1-null was slightly more frequent in women. The frequencies coincide with another study carried out in Caucasian control populations (35). Considering the relationship between GSTT1-null and toxicity, it is possible that this loss is caused by high toxicity in woman treated with the 5-Fu/CPT-11 schedules. In this sense, it has been observed that plasma concentrations of CPT-11 and its three metabolites (SN-38, SN-38G and APC) were in general, lower in males compared with females (36). However, the toxicity was independent of the gender and the relationship with the GSTT1-null was maintained after the $L R$ test.

The toxicity observed as the result of the loss of this gene is a new factor to be considered when this treatment schedule is administered to metastatic CRC patients.

Several new schemes of adjuvant therapy exist in advanced CRC patients. The pattern of inherited enzyme variants may become an additional factor to consider in deciding on the best course of therapy. The present study should be considered a preliminary finding until it has been validated in a larger study.

\section{References}

1. Braun AH, Achterrath W, Wilke H, et al: New systemic frontline treatment for metastatic colorectal carcinoma. Review. Cancer 100: 1558-1577, 2004

2. Folprecht $\mathrm{G}$ and Kohne $\mathrm{CH}$ : The role of new agents in the treatment of colorectal cancer. Review. Oncology 66: 1-17, 2004.

3. Douillard JY, Cunningham D, Roth AD, et al: Irinotecan combined with 5-fluorouracil compared with fluorouracil alone as first-line treatment for metastatic colorectal cancer: a multicentre randomized trial. Lancet 355: 1041-1047, 2000.

4. Saltz LB, Cox JV, Blanke C, et al: Irinotecan plus 5-fluorouracil and leucovorin for metastatic colorectal cancer. Irinotecan Study Group. New Engl J Med 343: 905-914, 2000.

5. Innocenti F, Undevia SD, Iyer L, et al: Genetic variants in the UDP-glucuronosyltransferase $1 \mathrm{~A} 1$ gene predict the risk of severe neutropenia of irinotecan. J Clin Oncol 22: 1382-1388, 2004.

6. Gupta E, Lestingi T, Mick R, et al: Metabolic fate of irinotecan in humans: correlation of glucuronidation with diarrhea. Cancer Res 54: 3723-3725, 1994.
7. Becouarn Y, Gamelin E, Coudert B, et al: Randomized multicenter phase II study comparing a combination of fluorouracil and folinic acid and alternating irinotecan and oxaliplatin with oxaliplatin and irinotecan in fluorouracil-pretreated metastatic colorectal cancer patients. J Clin Oncol 19: 4195-4201, 2001.

8. Yoshimatsu K, Kato H, Ishibashi K, et al: Second-line chemotherapy with low-dose CPT-11 and cisplatin for colorectal cancer resistant to 5-FU-based chemotherapy. Cancer Chemother Pharmacol 52: 465-468, 2003.

9. Coles B, Nowell S, MacLeod S, et al: The role of human glutathione S-transferases (hGSTs) in the detoxification of the food-derived carcinogen metabolite $\mathrm{N}$-acetoxy-PhIP, and the effect of a polymorphism in hGSTA1 on colorectal cancer risk. Mutat Res 482: 3-10, 2001.

10. Sweeney C, Coles BF, Nowell S, et al: Novel markers of susceptibility to carcinogens in diet: associations with colorectal cancer. Toxicology 181-182: 83-87, 2002.

11. Guy CA, Hoogendoorn B, Smith SK, et al: Promoter polymorphisms in glutathione-S-transferase genes affect transcription. Pharmacogenetics 14: 45-51, 2004.

12. Wang L, Groves MJ, Hepburn MD, et al: Glutathione Stransferase enzyme expression in hematopoietic cell lines implies a differential protective role for $\mathrm{T} 1$ and $\mathrm{A} 1$ isoenzymes in erythroid and for M1 in lymphoid lineages. Haematologica 85: 573-579, 2000.

13. Norppa H: Genetic susceptibility, biomarker responses and cancer. Mutat Res 544: 339-348, 2003.

14. Berhane K, Widersten M, Engstrom A, et al: Detoxication of base propenals and other alpha, beta-unsaturated aldehyde products of radical reactions and lipid peroxidation by human glutathione transferases. Proc Natl Acad Sci USA 91: 1480-1484, 1994.

15. Rowe JD, Nieves E and Listowsky I: Subunit diversity and tissue distribution of human glutathione S-transferases: interpretations based on electrospray ionization-MS and peptide sequence-specific antisera. Biochem J 325: 481-486, 1997.

16. Rebbeck TR: Molecular epidemiology of the human glutathione S-transferase genotypes GSTM1 and GSTT1 in cancer susceptibility. Cancer Epidemiol Biomarkers Prev 6: 733-743, 1997.

17. Reszka E, Wasowicz W, Rydzynski K, et al: Glutathione Stransferase M1 and P1 metabolic polymorphism and lung cancer predisposition. Neoplasma 50: 357-362, 2003.

18. Harries LW, Stubbins MJ, Forman D, et al: Identification of genetic polymorphisms at the glutathione S-transferase Pi locus and association with susceptibility to bladder, testicular and prostate cancer. Carcinogenesis 18: 641-644, 1997.

19. Kim JW, Lee CG, Park YG, et al: Combined analysis of germline polymorphisms of p53, GSTM1, GSTT1, CYP1A1 and CYP2E1: relation to the incidence rate of cervical carcinoma. Cancer 88: 2082-2091, 2000.

20. Monaghan G, Ryan M, Seddon R, et al: Genetic variation in bilirubin UPD-glucuronosyltransferase gene promoter and Gilbert's syndrome. Lancet 347: 578-581, 1996.

21. Saltz LB, Kanowitz J, Kemeny NE, et al: Phase I clinical and pharmacokinetic study of irinotecan, fluorouracil and leucovorin in patients with advanced solid tumors. J Clin Oncol 14: 2959-2967, 1996.

22. Masuda N, Kudoh S and Fukuoka M: Irinotecan (CPT-11): pharmacology and clinical application. Crit Rev Oncol Hematol 24: 3-26, 1996.

23. Rowinsky EK, Kaufmann SH, Baker SD, et al: A phase I and pharmacological study of topotecan infused over 30 min for five days in patients with refractory acute leukemia. Clin Cancer Res 2: 1921-1930. 1996

24. Shimada Y, Yashiro M, Wakui A, et al: Phase II study of CPT-11, a new camptothecin derivative, in metastatic colorectal cancer: CPT-11 Gastrointestinal Cancer Study Group. J Clin Oncol 11: 909-913, 1993.

25. Voso MT, D'Aló F, Putzulu R, et al: Negative prognostic value of glutathione S-transferase (GSTM1 and GSTT1) deletions in adult acute myeloid leukemia. Blood 100: 2703-2707, 2002.

26. Naoe T, Tagawa Y, Kiyoi H, et al: Prognostic significance of the null genotype of glutathione S-transferase-T1 in patients with acute myeloid leukemia: increased early death after chemotherapy. Leukemia 16: 203-208, 2002.

27. Davies SM, Robison LL, Buckley JD, et al: Glutathione Stransferase polymorphisms and outcome of chemotherapy in childhood acute myeloid leukemia. J Clin Oncol 19: 1279-1287, 2001 . 
28. Sweeney C, Nazar-Stewart V, Stapleton PL, et al: Glutathione S-transferase M1, T1 and P1 polymorphisms and survival among lung cancer patients. Cancer Epidemiol Biomarkers Prev 12: $527-533,2003$

29. Norppa H, Hirvonen A, Jarventaus H, et al: Role of GSTT1 and GSTM1 genotype in determining individual sensitivity to sister chromatid exchange induction by diepoxybutane in cultured human lymphocytes. Carcinogenesis 16: 1261-1264, 1995.

30. Landi S, Norppa H, Frenzilli G, et al: Individual sensitivity to cytogenetic effects of 1,2:3,4-diepoxybutane in cultured human lymphocytes: influence of glutathione s-transferase M1, P1 and T1 genotypes. Pharmacogenetics 8: 461-471, 1998.

31. Xu X, Wiencke JK, Niu T, et al: Benzene exposure, glutathione-s-transferase theta homozygous deletion, and sister chromatid exchanges. Am J Ind Med 33: 157-163, 1998.

32. Loktionov A, Watson MA, Gunter M, et al: Glutathione-Stransferase gene polymorphisms in colorectal cancer patients: interaction between GSTM1 and GSTM3 allele variants as a risk-modulating factor. Carcinogenesis 22: 1053-1060, 2001.
33. Deakin M, Elder J, Hendrickse C, et al: Glutathione S-transferase GSTT1 genotypes and susceptibility to cancer: studies of interactions with GSTM1 in lung, oral, gastric and colorectal cancers. Carcinogenesis 17: 881-884, 1996.

34. Chenevix-Trench G, Young J, Coggan M, et al: Glutathione S-transferase M1 and T1 polymorphisms: susceptibility to colon cancer and age of onset. Carcinogenesis 16: 1655-1657, 1995.

35. Garte S, Gaspari L, Alexandrie AK, et al: Metabolic gene polymorphism frequencies in control populations. Cancer Epidemiol Biomarkers Prev 10: 1239-1248, 2001.

36. Slatter JG, Schaaf LJ, Sams JP, et al: Pharmacokinetics, metabolism and excretion of irinotecan (CPT-11) following I.V. infusion of $[(14) C] C P T-11$ in cancer patients. Drug Metab Dispos 28: 423-433, 2000. 